\title{
Asimetrías de poder y el ejercicio de la autoridad en el trabajo doméstico pagado
}

\section{Power asymmetries and the exercise of authority in paid domestic work}

\author{
Rosario Fernández Ossandón \\ (D) https://orcid.org/0000-0003-3724-8678 \\ Instituto de Estudios Avanzados, Universidad de Santiago de Chile, Chile \\ Centro Núcleo Milenio Autoridad y Asimetrías de Poder \\ rosario.fernandez@usach.cl
}

Recepción: 21/01/21

Aprobación: 03/05/21

Publicación: 19/08/21

\begin{abstract}
The present article aims to stress the assumptions underlying the notion of power in studies on paid domestic work and proposes to include an empirical analysis of the management of power asymmetries through the exercise of authority. The methodology of the article bases on the review and analysis of theoretical and empirical texts on the notions of power and authority in gender studies in general, and studies on domestic work in particular, in particular emphasizing the Chilean context. The analysis shows the relevance of stressing the notion of power as domination and makes a proposal for the study of the exercise of authority on the basis of empirical investigation on the affective and intimate relationships between employers and domestic workers.
\end{abstract}

Key words: domestic work, power, exercise of authority, Chile.

Resumen: El presente artículo tiene como objetivo tensionar los supuestos que subyacen a la noción de poder en los estudios sobre el trabajo doméstico pagado y propone incluir un análisis empírico de la gestión de las asimetrías de poder a través del ejercicio de la autoridad. La metodología del artículo se basa en una revisión y análisis de textos teóricos y empíricos alrededor de las nociones de poder y autoridad en los estudios de género en general y los estudios sobre trabajo doméstico en particular, poniendo especial atención a la producción en Chile. El análisis da cuenta de la relevancia de tensionar la noción de poder como dominación y hace una propuesta para el estudio del ejercicio de la autoridad a partir de la investigación empírica de las relaciones afectivas e íntimas entre empleadoras y trabajadoras domésticas.

Palabras clave: trabajo doméstico, poder, ejercicio de la autoridad, Chile. 


\section{Introducción $^{1}$}

El trabajo doméstico constituye un nudo histórico de la teoría, de la política y práctica feminista, y es objeto de investigaciones respecto al patriarcado, el valor del trabajo reproductivo (Federici, 2012) y la relación de las mujeres con la política (Pateman, 1988). Pero especial atención ha adquirido el trabajo doméstico pagado como objeto para comprender las relaciones de poder articuladas por elementos estructurales e históricos en cuanto a las diferencias de género, raciales, étnicas y de clase social. Planteamos que en los estudios sobre trabajo doméstico ha predominado una definición de poder basada en las nociones de dominación y opresión, una herencia que también se encuentra en las teorías de género y feministas que comienzan a cuestionarse con el feminismo posestructuralista, posmoderno e interseccional, pero que no desaparece, influyendo en las formas en que estudiamos y comprendemos las relaciones de género. Si bien estos principios han sido cruciales para denunciar las relaciones de subordinación y las experiencias de las propias trabajadoras en agenciar y resistir dichas relaciones, consideramos que no logran dar cuenta de otras dinámicas de gestión de las asimetrías de poder, aquellas que no necesariamente responden a lógicas de dominación/subordinación o mando/obediencia. Argumentamos que la adopción de una noción de poder como forma de dominación y opresión es producto de cómo los estudios de género y feministas han privilegiado esta definición del poder y han descuidado otra categoría que creemos puede entregarnos luces sobre la gestión de las asimetrías de poder: el ejercicio de la autoridad. El objetivo del presente artículo es tensionar los supuestos respecto al poder en los estudios sobre este trabajo, para proponer que, además del poder, es preciso incluir un análisis empírico de la gestión de las asimetrías de poder a través del ejercicio de la autoridad, atendiendo a su dimensión íntima y afectiva.

El trabajo doméstico pagado es un objeto relevante para estudiar no sólo el poder, sino el ejercicio de la autoridad como herramienta de análisis. Su ambiguo espacio "entre medio" (Saldaña, 2014), que vincula la intimidad y

1 Este texto es el resultado del estudio empírico "Diferencia y figuras de autoridad femenina. Análisis a partir del trabajo doméstico pagado y el giro afectivo", agradecimientos a la Vicerrectoría de Investigación, Desarrollo e Innovación de la Universidad de Santiago de Chile (POSTDOC_DICYT, Código 031894_AK_MIL) por su financiamiento, y es también resultado del análisis teórico del proyecto "El ejercicio de la autoridad en las relaciones entre mujeres. Aproximaciones sociológicas a partir del estudio del trabajo doméstico pagado", Fondecyt Postdoctoral núm. 3210057, financiado por la Agencia Nacional de Investigación y Desarrollo de Chile (2021-2024). 
los afectos a relaciones contractuales, funciona como bisagra para configurar relaciones de poder en el contexto familiar y en tareas de cuidados y servicio (Gutiérrez, 2010). Esta práctica de "servir a otros" (Rossi y Campanella, 2018) nos convoca a pensar la gestión de asimetrías de poder en un espacio "íntimo" y que se traduce, muchas veces, en diversas y ambiguas estrategias de proximidad y distancia, de intimidad y diferenciación, de negociación y resistencia. En este artículo veremos que el ejercicio de la autoridad es una entrada analítica crucial para entender la gestión de las asimetrías de poder de forma situada y relacional, tensionando la noción jerárquica, binaria y dicotómica de la autoridad, en las relaciones íntimas afectivas, contractuales entre empleadoras y trabajadoras domésticas.

La metodología del artículo se basa en una revisión y análisis de textos teóricos y empíricos sobre las nociones de poder y autoridad en los estudios de género en general -siguiendo las propuestas de revisión de De la Fuente (2015) y Allen (2016) - y los estudios sobre trabajo doméstico en particular, poniendo especial atención a la producción en Chile, y en la presentación de una propuesta de investigación cuyo foco es el ejercicio de la autoridad entre empleadoras y trabajadoras domésticas. El caso chileno es relevante para dar luces respecto a lo que se ha denominado crisis de los cuidados. Para el caso en cuestión, dicha "crisis" muestra nudos específicos sobre cómo se experimentan las necesidades de cuidados en las clases acomodadas. Como indican otros estudios, la crisis es sentida como la ausencia de soluciones adecuadas a las necesidades de cuidado de la sociedad (Acosta, 2015) y producto del "problema de la niñera" (Staab y Maher, 2005), lo cual se refiere a la percepción entre las empleadoras de que hoy existiría una alta demanda de trabajadoras y, a la vez, una falta de mujeres dispuestas a desempeñarse como trabajadoras domésticas puertas adentro. Sin embargo, y como he señalado en otros escritos (Fernández, 2018a y 2018b), esta "demanda" o "crisis" tiene que ver más con la crisis de la familia -como referente nacional-y con las expectativas históricas de las empleadoras respecto a que las trabajadoras del hogar desempeñen sus funciones con amor, devoción y una actitud servil, que con un problema práctico relacionado con las condiciones de demanda/ oferta del trabajo doméstico. Esto hace interesante al caso chileno para el debate internacional sobre la crisis de los cuidados y otras formas de gestionar el poder en el contexto del deseo y nostalgia por la servidumbre y la crianza de niños/as (Canevaro, 2014). El caso chileno también es relevante porque, por un lado, muestra continuidades respecto a este legado colonial de la servidumbre al igual que otros países en América Latina (Saldaña, 2014; Rossi y Campanella, 2018), y, por otro, muestra transformaciones culturales 
y normativas en cuanto al ideal de relaciones más horizontales y de respeto entre sujetos diferentes (Araujo y Martuccelli, 2012). Esta simultaneidad hace que el caso chileno represente un aporte al estudio sobre el trabajo doméstico en un mundo global que requiere complejizar las formas de analizar el poder y su gestión en lazos afectivos e íntimos.

A continuación, explicaremos el foco de los estudios de género y feministas en las relaciones de poder y su influencia en los estudios sobre trabajo doméstico, en los cuales muchas veces se asume una noción de poder basada en relaciones de dominación/subordinación. Luego, a la luz de investigaciones recientes para el caso chileno y siguiendo la propuesta de Araujo (2020) respecto al estudio empírico de la autoridad, señalamos que el ejercicio de la autoridad como un problema sociológico relacional y situado es una propuesta de investigación necesaria para entender la gestión de las asimetrías de poder en un trabajo marcado por la intimidad y la afectividad.

\section{El poder en los estudios de género, feministas y sobre trabajo doméstico pagado}

\section{Elpoder en los estudios de género y feministas: el problema de la dominación}

Una parte importante de la sociología del género y de los estudios sobre el trabajo doméstico han concentrado su análisis en las relaciones de poder, la noción de autoridad patriarcal y la configuración de lugares de resistencia por parte de mujeres, inmigrantes, mujeres racializadas y mujeres de clase trabajadora, y han puesto menos atención a la noción de autoridad (Hanrahan y Antony, 2005). ${ }^{2}$ El protagonismo de la noción de poder se ha asumido muchas veces como una forma de gestión de las asimetrías a través de relaciones jerárquicas, de dominación y dicotómicas.

Siguiendo la lectura de Allen (2016), se pueden distinguir tres formas de concebir al poder en la teoría feminista y en la sociología del género: 1) el poder como recurso; 2) el poder como dominación; y 3) el poder como

2 Los trabajos de Hanna Fenichel Pitkin (The Concept of Representation; Fortune Is a Woman: Gender and Politics in the Thought of Niccolo Machiavelli; The Attack of the Blob: Hannah Arendt's Concept of "the Social") proponen hacer dialogar nociones de poder y autoridad. Sin embargo, dichos trabajos no realizan un estudio empírico del ejercicio de la autoridad. 
empoderamiento. ${ }^{3}$ Las tres concepciones, de acuerdo con la autora, comparten dos tensiones clave que, argumentamos, se vinculan con la pregunta sobre la autoridad sin incorporar explícitamente la autoridad como objeto de investigación. Primero, la supuesta diferencia entre el "poder-sobre" y el "poder-para", es decir, entre una concepción weberiana del poder como la capacidad de hacer a otros actuar según su voluntad y una concepción hobbesiana del poder como la capacidad de actuar (esto se ve especialmente en aquellas que se enfocan en la noción de agencia) (McNay, 2016). La sociología del género ha trabajado con diversas propuestas teóricas -desde la noción de patriarcado, de género y de interseccionalidad- para entrar desde ambos lados del poder (sobre y para). No obstante, si bien la mayoría de los autores reconocen ambos lugares, terminan enfocándose en el estudio de uno de ellos, ya sea en el poder sobre (como dominación) o el poder para (como agencia). Segundo, la distinción entre poder como acción y poder como eje estructural. El primero estaría enfocado en la capacidad individual de los actores de actuar (en nociones como resistencia o empoderamiento de las mujeres) y el segundo en los sistemas y matrices estructurales del poder generando una mirada relacional, social e histórica del poder.

$\mathrm{Al}$ retomar las tres concepciones del poder que define Allen (2016), la autora señala que en el caso de la definición del poder como recurso -especialmente evidente en trabajos inspirados en la teoría liberal del poderpredomina una noción de poder-para; es decir, el poder como un bien social (Okin, 1989) o como una capacidad (Nussbaum, 1999) que se encuentra distribuido desigualmente y que requeriría de una redistribución equitativa para que las mujeres puedan actuar en el mundo. Aquí el poder-para de las mujeres implicaría que ellas no tienen acceso a recursos, sin necesariamente generar una reflexión del poder de las mujeres sobre otros sujetos, reproduciendo una noción estática y aislada del contexto social en donde ocurren las relaciones de poder (de la Fuente, 2015).

En la concepción del poder como dominación, según cada postura teórica, puede tomar la forma de opresión, sujeción o sumisión, nociones que tienen como supuesto una relación de poder-sobre que es injusta o ilegítima. Esta perspectiva se observa claramente en los trabajos de la segunda ola y en el feminismo radical respecto a la noción de patriarcado como un sistema de

3 Estas tres concepciones funcionan como una forma para clasificar los usos de la noción de poder, pero no logran abarcar la totalidad de los usos ni pretende, en este artículo, representar una versión homogénea de los estudios sobre el poder. Por ejemplo, de la Fuente (2015) también trabaja con una tipología donde desarrolla cuatro formas de clasificar los usos del poder en la teoría feminista. 
dominación masculino (Amorós, 2005) y la familia como eje de la autoridad patriarcal (Millett, 1970). Pero también se ve reflejada en otras pensadoras y posturas teóricas. Por ejemplo, para de Beauvoir (1974), la opresión de las mujeres se entiende por la interpelación a ser el Otro de la cultura, sujetos no para sí sino para otros, limitando su libertad. Young (1992), Ahmed (2006) y Oksala (2016) se enfocan en el cuerpo y en la experiencia de opresión de las mujeres para entender mejor cómo se estructura la opresión, pero también cómo se resiste a ella. Otros ejemplos son los usos de la dicotomía dominación/subordinación, en especial en los trabajos de MacKinnon (1989), quien señala que la diferencia sexual es efecto de la dominación masculina, o en los de Frye (1983), que ve al poder en términos de acceso total versus ser accesible incondicionalmente. Por otro lado, la figura de la opresión también ha sido formulada desde una perspectiva del feminismo socialista, como en los trabajos de Firestone (1970), que sugieren que el género, como la clase, sería un sistema de opresión que funciona extrayendo el plus valor de la fuerza de trabajo reproductiva y donde la familia sería una institución de opresión hacia las mujeres (Millett, 1970; Delphy, 1982). Desde aproximaciones interseccionales se ha teorizado sobre el funcionamiento interconectado de distintas formas de opresión: de clase, género, sexualidad, raza, etnia, etcétera. El Combahee River Collective usó la noción de "interlocking systems of oppression” (CRC, 1981), mientras que King (1988) analiza las nociones de "multiple jeopardy and multiple consciousness". La interseccionalidad (Crenshaw, 1991) ha sido una noción importante para considerar las formas interconectadas de las experiencias de opresión, así como también la noción "interlocking systems of oppression" que intenta dar cuenta del aspecto más sistémico o estructural (Hill Collins, 2000). Estas perspectivas, junto con teorías feministas poscoloniales (Mohanty, 2003), decoloniales y de Abya Yala (Gargallo, 2014), se preguntan por el poder entre mujeres, el poder que ejercen unas mujeres con otras; sin embargo, en su análisis persiste un foco en la noción del patriarcado y en la experiencia de aquellas mujeres oprimidas por otras, en las consecuencias de ese ejercicio del poder, y no necesariamente centrando su análisis en cómo opera en concreto el poder-sobre. Por su parte, el feminismo posestructuralista se inspira en una noción del poder como un conjunto de relaciones de fuerza que emergen en cada interacción social y son parte del cuerpo social. El poder no estaría concentrado en un lugar, es capilar y local, estaría distribuido en el cuerpo social, aunque también puede funcionar en términos represivos, pero opera principalmente en la sujeción de los sujetos: la dominación funciona como una forma de creación de 
sujetos (Butler, 1990). Aquí también podemos situar los trabajos más contemporáneos influidos por el posestructuralismo, que desarrollan la noción de agencia como capacidad/poder de resistencia frente a lógicas de dominación (Lloyd, 2005; Allen, 2018).

En estas concepciones del poder como dominación, el poder-sobre está acentuado en las relaciones, ya sea en tanto el poder opresivo o de sujeción, especialmente desde lo masculino a lo femenino. Se otorga menos atención al ejercicio de autoridad como categorías de análisis y la comprensión de su ejercicio de las mujeres con otras mujeres. Si bien los aportes del feminismo interseccional, decolonial y poscolonial son importantes para mirar las relaciones entre mujeres, siguen acentuando la dominación como categoría a priori y, como consecuencia, requiriendo investigaciones empíricas respecto a cómo ocurre y se gestiona dicho poder-sobre (Hill Collins y Bilge, 2016).

Finalmente, la concepción del poder como empoderamiento -muy evidente en los trabajos sobre la diferencia sexual o sobre los cuidadostiende a acentuar una noción del poder-para; es decir, el poder como capacidad para la transformación personal y social, el poder desde dentro y el poder para una misma (Hoagland y Frye, 2000; Irigaray, 2009; León, 1997), dejando menos atención al poder-sobre.

Argumentamos que cuando se usan las tres concepciones del poder (el poder como recurso, como dominación y como empoderamiento) para comprender casos específicos de relaciones de asimetrías de poder, mantiene un protagonismo el eje del poder-sobre el cual es catalogado como injusto y estructural; una predominancia de la noción del poder ligada a la dominación y opresión; una herencia estructuralista de los conceptos de dominación y subordinación a partir de las teorías marxistas y del lenguaje; una herencia top-down, causa-efecto y jerárquica de la dominación (el género, la sexualidad, la clase y la raza serían estructuras que generan subjetividades y experiencias de subordinación e inferiorización); y una herencia binaria del poder basada en los binomios dominador/subordinadas y hombre/mujer, donde la agencia de los sujetos es comprendida como estrategias de sobrevivencia, y las luchas políticas redistributivas y por el reconocimiento se justifican por ser luchas de resistencia a esa dominación (Fraser y Butler, 2017; de la Fuente, 2015). Veamos cómo se refleja esto en los estudios sobre trabajo doméstico pagado en general y en particular para el caso chileno. 


\section{Trabajo doméstico pagado: poder, dominación y resistencia}

El trabajo reproductivo y doméstico (pagado y no pagado) ha sido un objeto importante dentro de la sociología del género y la teoría feminista. Sus estudios se inician con el debate sobre el valor del trabajo reproductivo en Estados Unidos en las décadas de 1960 y 1970, especialmente influidos por la segunda ola feminista. En su discusión con las teorías marxistas sobre el valor, el trabajo y la comodificación de éste, los primeros estudios exploraron la relación entre trabajo productivo y reproductivo usando teorías del desarrollo y la opresión de las mujeres en un modo de producción capitalista (Seccombe, 1974; Dalla Costa y James, 1972). Para el caso latinoamericano, el trabajo doméstico es analizado desde la década de 1970, influido por las teorías sobre la dependencia y la modernización (Safiotti,1978; Souza, 1980; Jelin, 1976). Estas primeras reflexiones se centraron en las condiciones materiales y económicas del trabajo doméstico, en particular en su importancia en las relaciones de clase y la formación de jerarquía racial y étnica en la región, a través de los procesos de migración campo-ciudad y la entrada de mujeres pobres, indígenas y racializadas en los hogares de clase media como trabajadoras domésticas (Tinsman, 1992). En ambas trayectorias, tanto la estadounidense como la latinoamericana, se destaca una comprensión de las relaciones de género como relaciones de poder basadas en la dominación patriarcal, argumentando que dicha dominación opera de forma particular en el espacio doméstico afectando negativamente a las trabajadoras domésticas mediante el "lazo de explotación" (Cox, 2006) entre trabajadoras y empleadoras.

Inclusive, en su interpretación respecto a la complejidad de las relaciones entre empleadoras y trabajadoras, una relación enraizada en patrones autoritarios, jerárquicos y patronales (Glenn, 1992; Chaney y García Castro, 1989), las complicidades y múltiples formas de negociación y resistencia que se producen también son vistas desde la óptica de la dominación, es decir, como estrategias de resistencia a relaciones de dominación. Si bien responden a estructuras de poder también, en las prácticas cotidianas, se visualizan formas diversas de gestionar y negociar las asimetrías de poder. Así lo manifiesta la publicación de Chaney y García Castro (1989), donde se describen las condiciones de explotación de este trabajo y las formas de opresión que sufren las trabajadoras, y las diversas maneras en que ellas negocian y resisten dicha situación, o los trabajos más contemporáneos sobre la crisis de los cuidados en Europa de Federici (2012), Precarias a la Deriva (2004) y Carrasquer Oto (2013), que señalan que la opresión de las mujeres en general, y de las trabajadoras domésticas en particular, es clave para la organización del capitalismo y el neoliberalismo. 
Esta discusión ha sido retomada por estudios internacionales sobre el trabajo doméstico y la migración, problematizando su articulación con la división internacional del trabajo de cuidado y la feminización del trabajo doméstico (Anderson, 2000; Chang, 2000; Herrera, 2005; Gutiérrez, 2010). Estos estudios también describen -así como lo hicieron aquellos en las décadas de 1980 y 1990- las relaciones globales de poder desde la óptica de la dominación y la opresión, exponiendo cómo las trabajadoras desafían las relaciones de dominación al interior de los hogares (Canevaro, 2014; Stefoni y Fernández, 2011).

Sin embargo, en los últimos años el interés por las relaciones íntimas y ambiguas entre empleadoras y trabajadoras, y la reproducción de las relaciones de poder basadas en el género, la raza y la clase, se convirtió en un foco importante de los estudios regionales (Canevaro, 2014; Bernardino-Costa, 2015; Casanova, 2019; Cordenonsi Bonez y Brites, 2020) e internacionales (Gutiérrez y Brites, 2014), que entendieron la imposibilidad de separar a los espacios íntimos del dominio público. El foco en las relaciones entre empleadoras y trabajadoras, así como la continua preocupación por destacar la agencia de éstas en un trabajo marcado por el "lazo de explotación" (Cox, 2006), evidencian las limitaciones de la óptica del poder como dominación, mostrando una deuda por estudiar empíricamente el ejercicio de la autoridad para la gestión de las asimetrías de poder en un trabajo afectivo e íntimo. Retomaremos más adelante esta línea de investigación y su vinculación con el estudio del ejercicio de la autoridad como una entrada teórico-empírica relevante para el análisis de la gestión del poder hoy.

En Chile, los estudios sobre trabajo doméstico surgieron a fines de la década de 1980 (Gálvez y Todaro, 1985, 1987 y 1989; Moreno, 1989; Montecino, 1993; Hutchinson, 2006), concentrándose principalmente en las condiciones laborales y las formas de organización social y política. Es importante destacar el libro de Gálvez y Todaro (1987): Trabajo doméstico remunerado. Conceptos, hechos y datos, pues fue la primera publicación dedicada exclusivamente a describir las situaciones de las trabajadoras domésticas chilenas, luego de años de investigación sobre el tema que desarrollaron las dos investigadoras del Centro de Estudios de la Mujer de Chile y trabajadoras domésticas organizadas. En la primera parte de su investigación definieron el trabajo doméstico como práctica reproductiva y productiva, y en la segunda, proporcionaron información estadística de las condiciones laborales. Con la influencia del feminismo marxista y preocupadas por las demandas políticas de las trabajadoras, las autoras asumen una noción del poder como dominación, y su hipótesis sugiere que las relaciones de producción basadas en la 
existencia de actitudes serviles de las trabajadoras domésticas determinan el comportamiento y la conciencia de éstas y sus capacidades para organizarse.

Sin embargo, este tema no se desarrolló más hasta la década de 2000, cuando la literatura sobre el trabajo doméstico y la migración reapareció parcialmente en las ciencias sociales (Stefoni, 2002; Staab y Maher, 2006; Stefoni y Fernández, 2011; Arriagada y Todaro, 2012; Del Campo y Ruiz, 2013; Leiva, 2015; Fernández, 2018a, 2018b y 2019; Valenzuela y Mora, 2009; CONLACTRAHO, 2004) y analizó principalmente la entrada de inmigrantes peruanas al trabajo doméstico remunerado y la intersección entre la nacionalidad y la división por género del trabajo de cuidado. La mayoría de las investigaciones se ha enfocado sobre todo en la situación de la Región Metropolitana, dejando un vacío en el estudio sobre lo que pasa en el resto del país. A su vez, han puesto especial atención a las formas de dominación por género y nacionalidad que se reproducen al interior de este oficio.

El interés por la migración y el trabajo doméstico fue acompañado por una serie de estudios sobre la falta de apoyo a las tareas de cuidado por parte del Estado (Arriagada y Todaro, 2012; Acosta, 2015). Lo que se ha denominado crisis de cuidado en el caso chileno (Arriagada y Todaro, 2012) se refiere a la situación donde más mujeres de clase media y alta acceden al mercado laboral requiriendo apoyo en las tareas domésticas y de cuidado, además existe una población que envejece y hay una falta de servicios de cuidado por parte del Estado.

Esto ha revelado que el cuidado sigue estando principalmente a cargo de las mujeres en los hogares, por lo cual el trabajo doméstico remunerado se convierte en una estrategia clave para estas familias. En este contexto, muchas familias de clase alta en Chile prefieren emplear a una trabajadora doméstica, porque creen que sus hogares son el mejor lugar para el cuidado de sus seres queridos (Fernández, 2017); esta situación depende de encontrar una manera de administrar este espacio con la ayuda de las trabajadoras domésticas remuneradas.

Sin embargo, Staab y Maher (2005) hacen un quiebre en la interpretación del trabajo doméstico y sus formas de dominación (como lo venía haciendo la literatura anterior) y se preguntan por los significados y emociones que circulan respecto a los cuidados y la demanda de trabajadoras domésticas. Las autoras argumentan que entre los empleadores, en Chile, surge el "problema de la nana": la dificultad de encontrar buenas trabajadoras, quienes conocen su lugar subordinado y realizan sus deberes con gracia. Según los empleadores entrevistados por Staab y Maher (2005), las trabajadoras domésticas de hoy no son como solían ser: tienden a saber 
demasiado sobre sus derechos y no están dispuestas a hacer todo lo que se espera de ellas.

Otros estudios (Fernández, 2018b y 2019) no sólo confirman estas disonancias respecto a la "buena nana", sino también dan cuenta de una ambivalencia a ejercer el rol de "jefa” por parte de la empleadora, moviéndose desde la crítica a una noción de "patrona" y su ejercicio imperioso de la autoridad hasta la incomodidad respecto al lazo afectivo que supone dicha posición. En estos estudios, mujeres de entre 40 y 70 años critican a las empleadoras más jóvenes sobre la "falta de autoridad" respecto a sus trabajadoras, es decir, que no ejercen un mando claro y directo; en tanto, las empleadoras de entre 20 y 40 años critican a las generaciones mayores por su vínculo patronal con las trabajadoras.

A su vez, las trabajadoras entrevistadas en dichos estudios señalan, primero, una incomodidad en cuanto al trato vejatorio por parte de sus empleadoras; y segundo, destacan que si bien existen relaciones de explotación, éstas no siempre logran explicar otras formas en que se vinculan empleadoras y trabajadoras. La dirección de los asuntos domésticos y el apoyo emocional y psicológico a la familia que las emplea son algunos de los ámbitos donde ellas también ejercían cierta autoridad.

Denominamos a estas incomodidades -tanto de las empleadoras como de las trabajadoras- la crisis de la relación "patrona/nana”, ya que una perspectiva del poder como dominación (que más bien se enfocaría en la reproducción del estatus de clase y racial de las familias que emplean y las formas que las trabajadoras negocian y se resisten a esta dominación) no sería suficiente para responder a la pregunta por la gestión del poder en las relaciones afectivas e íntimas. La definición del poder como dominación limita la emergencia de otras lecturas sobre la gestión de las asimetrías de poder que abra la pregunta sociológica sobre el ejercicio de la autoridad que no considere la dominación como un a priori.

A la luz de estas indagaciones, vislumbramos un problema de investigación que al ser leído desde las relaciones de poder/dominación no logra describir la crisis y los nuevos ajustes sobre el ejercicio de la autoridad en los espacios familiares y en las relaciones afectivas e íntimas entre empleadoras y trabajadoras domésticas. Es importante analizar la autoridad en una sociedad que supone la crisis de la autoridad tradicional y la demanda de relaciones más horizontales y democráticas (Araujo, 2016), así como una crisis de la relación "patrona/nana" a partir de una perspectiva sociológica que observe su puesta en práctica, es decir, su ejercicio. 


\section{El ejercicio de la autoridad como propuesta analítica para el estudio empírico del trabajo doméstico pagado}

\section{Crisis de la relación "patrona/nana" o por qué es importante estudiar el ejercicio de la autoridad entre empleadoras y trabajadoras domésticas}

El poder -como categoría de análisis en los estudios de género sobre trabajo doméstico- puede ser complementado con una lectura sociológica sobre el ejercicio de la autoridad. A continuación presentamos debates y estudios recientes en Chile que revelan la importancia de investigar los roles empleadora/patrona y trabajadora y el ejercicio de la autoridad entre ellas en el contexto de la crisis de la autoridad, convirtiendo al caso chileno en una puerta de entrada para el estudio de este tema en otros contextos. Luego revisaremos la propuesta de investigación sobre el ejercicio de la autoridad (Araujo, 2020) en el trabajo doméstico que definimos como afectivo e íntimo.

El 13 de julio de 2020, Soledad Brinck -una mujer dueña de casa y profesional de la clase alta chilena- posteó el siguiente tuit:

Llevo 6 meses sin nana, el ahorro q he tenido es tan Grande q es inevitable plantearse un cambio de modelo. Me imagino $\mathrm{q} x$ distintas razones hay mucha gente pensando lo mismo y no sola\% en el plano doméstico [...] Claro, no sólo el sueldo, se gasta menos, te rompen menos cosas, la casa está mucho más limpia, lo único malo es que estoy agotada pero basta con comprar un par de robots equivalentes a uno o dos meses de sueldo de la nana y la pega se aliviana mucho [...] un grupo q está acostumbrado a un sueldo muy por sobre el mínimo, con muchas ventajas y poca capacitación o preparación para hacer otras cosas (Brinck 2020).

El tuit generó bastantes polémicas en las redes sociales, algunas personas apoyaron y otras no. Empezó a circular la imagen del libro Vivir sin nana, donde Michelle Reich invita a las lectoras a "liberarse de la nana" (Reich, 2017: 5), destacando que el mercado de trabajo de las asesoras ha cambiado y que la relación laboral que se establece ya no es la misma. Reich llama a dejar de ser "patronas" y sólo contratar servicios de limpieza. También aparecieron otras opiniones entre las empleadoras con frases como "yo no podría vivir sin mi nana”, mostrando la otra arista de la dependencia afectivo-posesiva (por el uso del "mi") con las trabajadoras.

Por su parte, organizaciones feministas alertaron sobre el carácter clasista y deshumanizador del tuit. Las propias trabajadoras domésticas desde el Sintracap y Anecap (sindicato y asociación, respectivamente, de trabajadoras) manifestaron en sus redes sociales, así como en entrevistas en 
diarios y radios nacionales, su repudio a los dichos de Brinck y relataron la difícil situación que viven hoy en el contexto de la pandemia y visibilizaron sus demandas a través de la campaña \#CuidaaquienteCuida. Las situaciones que denuncian evidencian su condición desigual en la sociedad en términos legales, culturales y políticos. Sin acceso al seguro de cesantía (hasta octubre de 2020), la obligación de quedarse en los hogares de trabajo, la prolongación de horarios y la mayor cantidad de tareas demandadas ha llevado, incluso, a afirmar la existencia de una condición de esclavitud y servidumbre (Cáceres, 2020), haciendo eco con la situación de muchas trabajadoras domésticas en América Latina durante la pandemia Covid19 (De Costa, 2020; Desimone y Guerrero, 2020; Acciari, 2020).

"Patrona" y "sirvienta/nana" se viralizaron en las redes, enunciando posiciones y roles profundamente enraizadas en las relaciones entre empleadoras y trabajadoras domésticas y en la historia de nuestro país referida a las relaciones de mando/obediencia, a los lugares de autoridad y las relaciones laborales que se establecen en el espacio doméstico (Valdés, 2006). Posiciones saturadas en términos normativos por el estatus que portan, su uso hoy se mezcla con nociones más modernas (jefa y trabajadora), pero siguen apareciendo y recordando una memoria compleja respecto al ejercicio de la autoridad y las relaciones de asimetrías de poder (Fernández, 2018b).

Estas situaciones se desarrollan en el contexto actual donde modelos de familia igualitarios conviven con modelos tradicionales, generando un "conservadurismo fracturado" (Martínez y Palacios, 2001) o un "tradicionalismo moderno" (Valdés y Valdés, 2005). A esto se le suman los procesos de individualización de la población en general y especialmente en el caso de las mujeres quienes se han convertido en protagonistas de las transformaciones de las definiciones, prácticas, discursos y patrones familiares (Araujo y Martuccelli, 2012). Este protagonismo de las mujeres se ve tanto en las empleadoras como en las trabajadoras, y convive con la persistencia de la necesidad de contratar a otras mujeres para la realización de los proyectos de las mujeres de clase media y acomodada, principalmente por la inexistencia de un Estado de bienestar que brinde soportes para los cuidados y la des-responsabilización masculina respecto a las tareas domésticas y parentales, incluso a pesar de los nuevos discursos sobre las parentalidades modernas (Acosta, 2015; Valdés, 2008; Gómez y Jiménez, 2015). La contratación de la "nana" es parte de los resabios o actualizaciones del modelo tradicional de la gestión de los hogares de clase media-acomodada (Montecino, 1993).

Si bien estudios en Chile demuestran una transformación en la gestión de las asimetrías de poder en el ámbito familiar y privado hacia un modelo 
más democrático de las relaciones de género, también se destaca que en dichas gestiones persisten modelos tradicionales e, incluso, patronales ligados a las nociones de "buenas" patronas y "buenas" nanas (Fernández, 2018b), que se manifiestan en las relaciones dentro de los hogares (Valdés, 2006). El tuit y los estudios sobre el tema (Staab y Maher, 2005 y 2006; Stefoni y Fernández, 2011; Fernández, 2018a) dan cuenta de que hoy existe una incomodidad tanto de las empleadoras como de las trabajadoras respecto a la relación laboral y afectiva entre ellas, relación que se encuentra en un estado paradojal. Como dichas investigaciones han analizado, por un lado, las empleadoras señalan no querer "nanas" o no poder vivir sin ellas, dos polos (el primero de carácter más "moderno" y el segundo más tradicional o dependiente) respecto a la incomodidad con esta relación y de ejercer el rol de empleadoras. Por el otro lado, las trabajadoras insisten en su lugar como tales a través de demandas y luchas en el ámbito político y legal, como también en su lugar de autoridad, es decir, la categoría trabajadoras las autoriza y legitima a ejercer su rol contractual y afectivo en sus empleos.

Lo paradojal de esta situación es que ambos actores reconocen que hay una relación de autoridad y que dicha autoridad no necesariamente la "posee" sólo una parte (se construye de manera relacional), pero no por ello saben cómo ejercer la autoridad en una relación que aún se construye dentro de los términos tradicionales-patronales (de explotación y del registro tradicional-afectuosa que supone) en un contexto de mayor demanda por vínculos y formas de relacionarnos más horizontales, democráticas y modernas (Araujo y Martuccelli, 2012). Esta paradoja se enmarca en una forma histórica de producción de la sociedad y de las familias propia del continente y del contexto chileno patronal, y que hoy se quiere plantear en términos modernos sin tener un registro sobre cómo hacerlo. Valdés (2008:54) señala que nos encontramos en:

[...] una sociedad sobre-familiarizada pero a la vez testigo de una institución fracturada y tensionada por nuevas expectativas de sus miembros en ausencia de dispositivos que faciliten la transición de un modelo a otro. Este tipo de fenómenos forman parte de estos síntomas de crisis y de malestar que se perciben desde dentro de las familias [...] ante los imperativos de la vida moderna y la debilidad de los sistemas de protección social.

Entonces, el problema no es sólo la reproducción de la lógica poder/ dominación, sino la pregunta sobre cómo se configura un vínculo contractual y afectivo y cómo se gestiona la autoridad en él. La relación entre empleadoras y trabajadoras arrastra esta historia y hoy se ve fuertemente afectada por las transformaciones en los modos en que se ejerce la autoridad y se 
gestionan las asimetrías en Chile. Este escenario - contradictorio y disonante- nos abre la pregunta sociológica sobre la reproducción de los hogares y la gobernanza que requiere, ya sea por el rol que cumplen las mujeres, las empleadoras y las trabajadoras domésticas, y las tensiones contemporáneas que se observan en las relaciones entre ellas en una sociedad que dice estar en una crisis de los modelos de autoridad (Araujo, 2016).

Dicha crisis se refiere a una falta de legitimidad, especialmente sobre la noción de autoridad tradicional de mando/obediencia en los espacios estatal, laboral y familiar, pero también a la falta de nuevos repertorios sobre cómo definir y ejercer otras formas de autoridad en el contexto de demandas por relaciones más horizontales y democráticas. Araujo (2016) sostiene que en la actualidad los actores sienten una cierta ambivalencia (entre el autoritarismo y las nociones modernas de autoridad) respecto al ejercicio de la autoridad en relaciones de asimetrías de poder. El problema con la autoridad hoy no sería su debilitamiento, sino la necesidad de comprender su ejercicio.

¿Cómo se recompone la relación entre empleadoras y trabajadoras ahora? ¿Cómo se ejerce la autoridad en esta relación en dicho contexto paradojal? Éstas son preguntas sin respuestas claras. Creemos que una aproximación al ejercicio de la autoridad entre empleadoras y trabajadoras domésticas es una herramienta analítica para indagar en estas preguntas.

\section{La autoridad en la teoría social}

La sociología y la filosofía política se han enfocado en analizar críticamente a la autoridad como el principio y el ejercicio del orden político, y sus formas de legitimidad a través de la obediencia y el reconocimiento (Weber, 1964; Arendt, 1996; Kojève, 2005). En esta trayectoria, la autoridad no es definida como el ejercicio de cualquier forma de poder, sino como una modalidad de poder que se produce en la relación mando/obediencia a través de la persuasión y argumentación, y no (necesariamente) en el uso de la fuerza (Arendt, 1996).

En la relación de mando/obediencia habría una jerarquía, pero se requiere tanto de quien la ejerce como de quien la recibe, ambos lados serían constitutivos de ella. La sociología ha tomado esta herencia entendiendo que el estudio de la autoridad necesita a su vez del estudio del poder y las asimetrías sociales; es decir, autoridad y poder son parte de los mecanismos de ordenamiento y funcionamiento de la sociedad. En sus inicios, la sociología definió a la autoridad en términos jerárquicos, referidos a la lógica 
obediencia/resistencia y en espacios públicos (estatal, institucional y labo$\mathrm{ral} /$ productivos) (Araujo, 2016). ${ }^{4}$

Durante el siglo XX, y especialmente por los efectos de las guerras en Europa, los trabajos sobre la autoridad se abocaron a la revisión crítica del autoritarismo, enfocándose en el problema de la dominación de los Estados sobre los ciudadanos (Foucault, 1999), el autoritarismo y la violencia (Arendt, 1996), y el problema del reconocimiento (Gadamer, 1977) y respecto a la obediencia (Adorno, 1965). Si bien dichos trabajos se centraron en la relación entre autoritarismo y autoridad, hacia mediados y fines de siglo concuerdan en la existencia de un debilitamiento de la figura de la autoridad autoritaria, en particular respecto a su legitimidad; incluso llegan a concluir que hoy nos encontramos frente a una crisis de la autoridad (Arendt, 1996). Tanto por su vinculación con el autoritarismo como por su su supuesto debilitamiento, el estudio sobre la autoridad fue perdiendo espacio dentro de la sociología y las ciencias sociales (Cohen, 2013), ganando predominancia las investigaciones sobre el poder, especialmente a partir de la influencia de las teorías posestructuralistas.

En el contexto latinoamericano ha predominado el estudio de la autoridad ligada al autoritarismo, sobre todo a causa de las dictaduras y regímenes dictatoriales entre 1960 y 1990 . Al estar instalada en una disputa valórica sobre su rol en la sociedad, ya sea como garante del orden o como forma de dominación y por estar basada en investigaciones desarrolladas principalmente en el contexto europeo, hoy en día vemos ciertos vacíos teóricos y metodológicos respecto al estudio sobre autoridad (Araujo, 2016). Planteamos que habría una escasez de investigaciones empíricas sobre el ejercicio de la autoridad desde el caso latinoamericano y chileno en particular, considerando el contexto de las transformaciones sociales de la modernidad (Araujo y Martuccelli, 2012). Esta falta se debe, en parte, a una definición normativa de la autoridad que la vincula al autoritarismo o como algo exclusivo del ámbito público y político.

En el caso chileno, la autoridad ha sido estudiada para comprender la cultura del autoritarismo (Brunner, 1981), pero hoy se ha establecido la

$4 \mathrm{Su}$ estudio se enfocó en analizar teóricamente su estatuto, su legitimidad y lugar en el funcionamiento de la sociedad, en especial respecto a la relación entre Estado y soberanos (en los trabajos de Weber) y su rol normativo en el mantenimiento del orden social (en los escritos de Durkheim), tema que ha sido central y transversal en los estudios posteriores sobre la autoridad. Los análisis de Weber han sido fundamentales y tuvieron una gran influencia dentro de la sociología para indagar en la legitimidad, la burocracia y el lugar de la tradición en la autoridad. 
percepción de que la autoridad se encuentra en una crisis de legitimidad, tanto en el espacio estatal y laboral como en el familiar. Sin embargo, de acuerdo con Araujo (2016), el problema no sería su debilitamiento o crisis, sino la necesidad de comprender su ejercicio. La autora avanza hacia el estudio de la autoridad entendiéndolo como un ejercicio relacional, situado e histórico; asimismo, ha investigado dicho ejercicio en el ámbito familiar, en las relaciones de pareja y en las relaciones con los/as hijos/as, enfocándose en las continuidades y transformaciones de las relaciones de género, dando cuenta de un protagonismo de las figuras femeninas de autoridad en el espacio familiar y extrafamiliar. Araujo (2016) propone una aproximación sociológica al ejercicio de la autoridad en el contexto de la simultánea existencia de lógicas tradicionales y modernas de concebir la autoridad y en uno de mayor demanda por expandir las nociones de ciudadanía, igualdad y democracia.

En este escenario, proponemos profundizar en los estudios sobre el ejercicio de la autoridad en el ámbito familiar y reproductivo, en especial en las relaciones entre mujeres, dada la laguna de investigaciones empíricas sobre el ejercicio de la autoridad entre mujeres tanto desde la sociología como desde los estudios de género. La noción de autoridad en la teoría de género y feminista ha tendido a ser conceptualizada en términos negativos, entendiéndose muchas veces como sinónimo del orden patriarcal o masculino (Amorós, 2005) o del autoritarismo (Hanrahan y Antony, 2005), y las propuestas se han dirigido a pensar cómo entender el poder de forma no autoritaria (Femenías 2000).

Respecto a los estudios concretos sobre la autoridad, se enfocan específicamente en figuras de autoridad femenina -como el lugar simbólico de la madre (Vera, 2016; Muraro, 1991) o de las diosas (Verdú, 2012) o el rol social de las profesoras y de las asistentes sociales (Illanes, 2006)-, o el estudio de mujeres "destacadas" -en posiciones de liderazgos en la esfera laboral y de la política institucional (Valenzuela 2019)-. Estos trabajos son importantes porque evidencian las dificultades para acceder a lugares de autoridad y lo complejo que es ejercerla. Por ejemplo, llegar a ser profesora de universidad y ejercer el oficio da cuenta de la persistencia de una cultura que le dice que ese no es su lugar (Hanrahan y Antony, 2005).

Dichos lugares de autoridad femenina suelen usar como soporte la tradición, conocimientos sobre lo doméstico y el cuerpo o el rol reproductivo para justificar su poder-sobre, su lugar de liderazgo y su estatus dentro de una comunidad. Los estudios citados tienden a considerar los lugares de autoridad femeninos como simbólicos, donde las mujeres adquieren poder, derechos, reconocimiento y legitimidad en un contexto o situación específica por 
cumplir roles o labores femeninas destacados. En ellos, la diferencia sexual es un factor explicativo de dichos lugares de reconocimiento, pero también muchas veces es un a priori sin necesariamente explicar cómo se ejerce esa autoridad.

\section{El estudio del ejercicio de la autoridad en las relaciones afectivas e intimas entre empleadoras y trabajadoras}

A continuación planteamos comprender los modos en que se gestionan las jerarquías y diferencias entre mujeres, y entre empleadoras y trabajadoras, para organizar lo doméstico a través de una lectura sobre el ejercicio de la autoridad, siguiendo la propuesta teórica y metodológica de Kathya Araujo, y la definición del trabajo doméstico como una práctica performativa afectiva e íntima (Fernández, 2018b).

De acuerdo con Araujo (2016:14-15):

(1)a autoridad es, pues, un fenómeno esencial para entender una sociedad porque participa en establecer las modalidades de gestión de las jerarquías, lo que constituye un componente indispensable para dar cuenta de las maneras en que una sociedad ha resuelto la cuestión del lazo social (Freud, 1999): esto es, las formas de convivencia, así como las modalidades de enlazamiento que la caracterizan.

¿Cómo se gestionan las asimetrías de poder en el ejercicio de la autoridad entre mujeres?, ¿qué lazo social se produce con el ejercicio de la autoridad entre empleadoras y trabajadoras domésticas en el ámbito familiar? Creemos que estas preguntas complementan los estudios sobre las relaciones de poder entre ellas, para profundizar en cómo efectivamente se gestionan las asimetrías y se construyen las relaciones laborales y afectivas. Consideramos que a las investigaciones sobre trabajo doméstico enfocadas en las relaciones de poder es necesario sumar estudios empíricos sobre el ejercicio de la autoridad en las relaciones entre empleadoras y trabajadoras domésticas. Es decir, estudios que comprendan los modos concretos en que se ejercen los roles de empleadoras y trabajadoras en un contexto y momento histórico determinado, relacional e interactivo (en función de las relaciones entre actores específicos y que se constituye en la interacción misma entre estos actores), y a través del uso de soportes y mecanismos específicos.

Este tipo de análisis nos permitirá, además, des-sustancializar la noción de autoridad. Así como Butler (1990) plantea la necesidad de comprender la producción variable de la identidad desmitificando su estatuto esencialista, también podemos comprender a la autoridad como una realización que se produce en su ejercicio, mostrando sus continuidades y su transformaciones. 
Alejándonos de una noción de la autoridad como un atributo dado de antemano e invariable, proponemos el ejercicio de la autoridad como una producción relacional y mutable.

A su vez, siguiendo los aportes de Gutiérrez (2010) sobre el carácter afectivo del trabajo doméstico, proponemos una definición de éste como un sitio performativo íntimo-político, porque no sólo implica realizar tareas específicas al mismo tiempo, sino que también es un trabajo que se encarna espacial y físicamente en un conjunto de prácticas donde las personas reproducen significados sociales y culturales a través de sus interacciones (Fernández, $2018 b$ ). Sugiero que no hay empleadoras y trabajadoras domésticas preexistentes, más bien se reproducen continuamente en las relaciones íntimas entre ellas haciendo referencia a una genealogía afectiva.

El trabajo doméstico se ve influido por afectos históricamente feminizados y racializados y, por tanto, posee un carácter performativo donde los afectos modulan las relaciones empleadora-trabajadora. Reconocer el carácter performativo, afectivo e íntimo nos permite comprender que la gestión de las asimetrías entre mujeres no sólo puede ser entendido como un ejercicio del poder como dominación, sino en una gestión plural y diversa del poder, siendo una de esas formas el ejercicio de la autoridad. Dicho ejercicio se despliega de forma relacional entre empleadoras y trabajadoras, y está marcado tanto por su contingencia como por su carácter histórico. Interesa indagar, entonces, cómo se ejerce la autoridad en las coordenadas de la afectividad y la intimidad en las relaciones entre empleadoras y trabajadoras y cómo este ejercicio puede reproducir o transformar las dinámicas de dominación, así como generar otras modulaciones del poder que no se reducen a la lógica emancipación versus dominación y que más bien muestran la simultaneidad y complejidad en que el poder -entre mujeres- opera.

Siguiendo el Enfoque Interactivo y Relacional de la Autoridad (EIRA) (Araujo, 2020), desarrollado por Kathya Araujo en el marco del Centro Núcleo Milenio Autoridad y Asimetrías de Poder (USACH-UDP, Chile), entendemos que el ejercicio de la autoridad supone actores, soportes y mecanismos concretos. El EIRA define actores como la participación de individuos que tienen una dotación de poder diferencial en cualquiera de las posiciones donde se despliega el ejercicio de autoridad, un poder de carácter social (relacionado a estereotipos, representaciones, jerarquías valóricas, etc.) y/o producido individualmente, pero que se genera en la relación e interacción con otros, en este caso entre empleadora y trabajadora. Por soporte, el modelo propone que los actores tienen a su disposición diferentes tipos de recursos que los apoyan o sostienen (como el prestigio social, investiduras simbólicas, 
atribuciones legales, grados atribuidos de confianza, relación con la verdad, etc.) el ejercicio de la autoridad.

Si bien estos recursos están disponibles, no siempre se constituyen en soportes, pues depende de cómo son percibidos y movilizados por los actores. Por mecanismos el modelo EIRA propone que el ejercicio de la autoridad supone una dimensión de ejecución o despliegue concreto que busca hacer posible que efectivamente se influyan u orienten las acciones o conductas de aquellos respecto de los cuales se ejerce la autoridad.

Para nuestro caso, significa pensar que la autoridad no es algo que portan las/los empleadores/as, sino una práctica social que se ejerce según los actores y su dotación de poder, cuyos soportes (como el vínculo emocional o los contratos de trabajo) y mecanismos concretos (como la definición de tareas y horarios, solicitudes de permisos, negociaciones de pagos por horas extras, apelación al carácter femenino de las tareas de cuidados, por ejemplo) pueden cambiar con el tiempo. A su vez, la producción y circulación de afectos en el ejercicio de la autoridad -como la rabia, la empatía, el asco, la vergüenza- pueden modificar o tensionar las formas en que se ejerce la autoridad entre empleadoras y trabajadoras. Para estudiar la gestión de las asimetrías de poder entre ellas, se podría incluir:

1) Los actores y cómo adquieren, pierden, comparten, transan, negocian o gestionan dotaciones de poder para definir las tareas y roles de cada actor, expectativas y responsabilidades, derechos y límites de cada uno. Además de las empleadoras y trabajadoras, otros actores son relevantes para pensar cómo se ejerce la autoridad, por ejemplo: abuelas, suegras, amistades, hijos/as, etc. Los afectos y el lazo afectivo entre los actores y las relaciones de intimidad entre ellos son importantes a considerar para analizar cómo se manifiesta el lugar material y simbólico que ocupa cada actor en prácticas concretas, así como dichos afectos reproducen o transforman lógicas de poder.

2) Indagar en los soportes y recursos simbólicos y materiales, como pueden ser los afectos y los vínculos de dependencia emocional entre empleadoras y trabajadoras, el conocimiento sobre la intimidad de la vida de las empleadoras/trabajadoras, el conocimiento sobre lo que a la otra le afecta o cómo puede reaccionar emocionalmente a las peticiones o demandas de la otra parte, las leyes o discursos públicos sobre el trabajo doméstico o de cuidados, la moral y las normas sociales vigentes sobre el rol de las mujeres y de los padres en la reproducción de los hogares y en la crianza, etc., para hacer circular el poder o tensionar las asimetrías.

3) Analizar los mecanismos concretos con los cuales se ejerce la autoridad, es decir, aquellas prácticas donde se definen o ponen límites, como 
puede ser la comunicación y el diálogo, el despido, la denuncia legal, la demanda de cariño o una disposición afectiva con la familia, apoyo especial en tareas de cuidado como la maternidad y la crianza, entre otras.

Estos tres elementos nos permiten pensar críticamente las incomodidades que hoy se presentan respecto a los roles de "empleadoras" y "nanas", así como las nuevas formas de gestionar y articular las relaciones de asimetrías de poder entre ellas sin dar por hecho la dominación y explotación como un a priori, sino como una de las formas en que las relaciones entre ellas se articulan y el rol que cumplen los afectos en dichas relaciones. Creemos que el estudio sobre la gestión del poder desde la perspectiva del modelo EIRA nos permite profundizar en el carácter relacional y performativo del poder, así como su gestión a través de registros afectivos e íntimos en las relaciones entre empleadoras y trabajadoras domésticas hoy. Entendiendo los afectos como pulsiones que circulan (Ahmed, 2006), podemos pensar que el ejercicio de la autoridad produce y hace circular afectos que pueden reproducir formas jerárquicas del poder como también habilitar y potenciar formas de gestión de las asimetrías que irrumpen y suscitan otras figuraciones posibles.

\section{Reflexiones finales}

Estudiar empíricamente el ejercicio de la autoridad entre empleadoras y trabajadoras domésticas tiene desafíos específicos. El primero es un desafío teórico, pues predominan los análisis enfocados en las relaciones de poder y el entendimiento del poder como una forma de dominación. El segundo desafío es empírico: el estudio sobre el ejercicio de la autoridad en el contexto de la crisis de la autoridad y la crisis de la relación "patrona/nana".

En este escenario, la figura de autoridad ha sido definida como el lugar del poder, el que domina, el que oprime, de carácter masculino, limitándose no sólo el estudio a la autoridad y su ejercicio (por mujeres, entre mujeres o la autoridad de hombres desde una perspectiva relacional), sino también al poder. Argumentamos que este escenario ha generado límites para pensar la autoridad como una práctica relacional, situada e histórica, es decir, como un fenómeno social, careciendo de estudios empíricos y de herramientas metodológicas para la investigación sobre el ejercicio de la autoridad.

A su vez, las tres definiciones de poder (como recurso, como dominación y como empoderamiento) presuponen la diferencia sexual como categoría de análisis, y los lugares de autoridad o liderazgo femeninos son observados positivamente (ya sea porque empodera a las mujeres o porque genera otro tipo de liderazgo) y presuponen una relación de asimetrías de poder entre 
hombres y mujeres (y no necesariamente entre mujeres). Mientras tanto, el poder como recurso y el poder como empoderamiento ponen mayor atención a la noción del poder-para, y la noción del poder como dominación se enfocaría en una noción del poder-sobre. Esto es un problema para el estudio del ejercicio de las relaciones de poder, pues para analizar el poder-sobre también habría que estudiar el poder-para, no se podrían separar. Ese sería un aporte del análisis del ejercicio de la autoridad entre mujeres: estudiar ambos aspectos del poder. Esto nos permitirá examinar ambos lados de éste a través del análisis de la gestión de las asimetrías de poder dentro del hogar en términos concretos, es decir, como un fenómeno social en el contexto de la crisis de la autoridad y de la relación "patrona/nana”, considerando su carácter relacional, situacional y sociohistórico, así como en su registro afectivo e íntimo.

El actual escenario de las transformaciones sociales de mayor demanda por relaciones horizontales y democráticas y de la crisis de la relación "patrona/nana" es provechoso para comprender el ejercicio de la autoridad en relaciones de asimetrías de poder. Los estudios empíricos sobre el ejercicio de la autoridad en las relaciones y lazos afectivos e íntimos entre empleadoras y trabajadoras domésticas se vuelven un campo relevante para las ciencias sociales, ya que pueden ofrecer nuevos escenarios interpretativos sobre el trabajo doméstico pagado y la gestión de las asimetrías de poder en él. Esperamos que a partir de los resultados empíricos del estudio 5 podamos complejizar el análisis sobre el ejercicio de la autoridad, destacando su carácter afectivo y el lugar e importancia del reconocimiento -elemento que la teoría feminista ha resaltado como fundamental en la reestructuración de las relaciones de poder (Butler, 1990; Fraser y Butler, 2017)- en dicho ejercicio.

\section{Referencias}

Acciari, Luoisa (2020), “'A trabalhadora doméstica também é um ser humano’: O direito a quarentena remunerada como novo privilégio social?”, en Reflex Pandemia. Disponible en: https://www.reflexpandemia.org/texto-5 [20 de junio de 2020].

Acosta, Elaine (2015), Cuidados en crisis. Mujeres migrantes hacia España y Chile, España: Universidad de Deusto.

Adorno, Theodor (1965), La personalidad autoritaria, Argentina: Proyección.

Ahmed, Sara (2006), Queer Phenomenology: Orientations, Objects, Others, Estados Unidos: Duke University Press.

5 "Diferencia y figuras de autoridad femenina. Análisis a partir del trabajo doméstico pagado y el giro afectivo”. Financiado por la Vicerrectoría de Investigación, Desarrollo e Innovación de la Universidad de Santiago de Chile. 
Allen, Amy (2016), "Feminist Perspectives on Power", en Zalta, Edward [ed.], The Stanford Encyclopedia of Philosophy, Estados Unidos: Stanford University Press.

Allen, Amy (2018), The power of feminist theory, Estados Unidos: Routledge.

Amorós, Celia (2005), “Dimensiones de poder en la teoría feminista”, en Revista Internacional de Filosofia Politica, núm. 25, México, Universidad Autónoma Metropolitana.

Anderson, Bridget (2000), Doing the dirty work?: the global politics of domestic labour, Reino Unido: Zed Books.

Araujo Kathya y Martuccelli, Danilo (2012), Desafios comunes. La sociedad chilena y sus individuos, tomo I, Chile: LOM.

Araujo, Kathya (2016), El miedo a los subordinados. Una teoría de la autoridad, Chile: LOM.

Araujo, Kathya (2020), Enfoque Interactivo y Relacional de la Autoridad (EIRA), Manuscrito en prensa, Chile.

Arendt, Hannah (1996), Entre el pasado y el futuro, ocho ejercicios sobre la reflexión politica, España: Península.

Arriagada, Irma y Todaro, Rosalba (2012), Global Care Chains: the role of Peruvian immigrants in the provision of care in Chile, República Dominicana: ONU Women.

Beauvoir, Simone de (1974), The Second Sex, Estados Unidos: Vintage Books.

Bernardino-Costa, Joaze (2015), "Decolonialidade e interseccionalidade emancipadora: a organização política das trabalhadoras domésticas no Brasil", en Sociedade e Estado, vol. 30, Brasil: Departamento de Sociologia da Universidade de Brasília.

Brinck, Soledad (2020), "tuit sobre ausencia de nana" [twitter], 13 de junio de 2020.

Brunner, José (1981), La cultura autoritaria en Chile, Chile: Facultad Latinoamericana de Ciencias Sociales.

Butler, Judith (1990), Gender Trouble: Feminism and the Subversion of Identity, Estados Unidos: Routledge.

Canevaro, Santiago (2014), "Afectos, saberes y proximidades en la gestión del cuidado de niños. Empleadas y empleadoras del servicio doméstico en Buenos Aires", en Durin, Séverine, de la O Martínez, María Eugenia y Bastos, Santiago [eds.], Trabajadoras en la Sombra. Dimensiones del servicio doméstico latinoamericano, México: Centro de Investigaciones y Estudios Superiores en Antropología Social.

Carrasquer Oto, Pilar (2013), "El redescubrimiento del trabajo de cuidados: algunas reflexiones desde la sociología”, en Cuadernos de Relaciones Laborales, núm. 2, España: Universidad Complutense de Madrid.

Casanova, Erynn (2019), Dust and Dignity: Domestic Employment in Contemporary Ecuador, Estados Unidos: Cornell University Press.

Cáceres, Daniela (2020), “\#CuidaAQuienTeCuida: la compleja situación que enfrentan las trabajadoras de casa particular en tiempos de pandemia”. Disponible en: https://www. elmostrador.cl/braga/2020/06/19/cuidaaquientecuida-la-compleja-situacion-queenfrentan-las-trabajadoras-de-casa-particular-en-tiempos-de-pandemia/ [19 de junio de 2020].

Chaney, Elsa y Garcia Castro, Mary (1989), Muchachas no more. Household workers in Latin America and the Caribbean, Estados Unidos: Temple University Press.

Chang, Grace (2000), Disposable Domestics Immigrant Women Workers in the Global Economy, Estados Unidos: South End Press.

Cohen, Yves (2013), Le siècle des chefs: Une histoire transnationale du commandement et de l'autorité (1890-1940), Francia: Éditions Amsterdam. 
Convergencia Revista de Ciencias Sociales, vol. 28, 2021, Universidad Autónoma del Estado de México

CONLACTRAHO [Latin American and Caribbean Confederation of Household Workers] (2004), Humanizando el trabajo doméstico: hacer visible lo invisible. La realidad de las trabajadoras del hogar en América Latina y el Caribe: Bolivia, Brasil, Costa Rica, Guatemala, México, Perú y República Dominicana, Chile: Alerce Talleres Gráficos.

Cordenonsi Bonez, Mateus y Brites, Jurema (2020), "O trabalho de cuidado no sindicato das trabalhadoras domésticas de Pelotas, RS”, en Século XXI: Revista de Ciências Sociais, vol. 9, núm. 3, Brasil: Centro de Ciências Sociais e Humanas da Universidade Federal de Santa Maria.

Cox, Rosie (2006), The Servant Problem: Domestic Employment in a Global Economy, Inglaterra: I.B. Tauris.

CRC [Combahee River Collective] (1981), “A Black Feminist Statement (1977)”, en Moraga, Cherríe y Anzaldua, Gloria [eds.], This Bridge Called My Back: Writings by Radical Women of Color, Estados Unidos: Kitchen Table y Women of Color Press.

Crenshaw, Kimberle (1991), "Mapping the Margins: Intersectionality, Identity Politics, and Violence Against Women of Color”, en Stanford Law Review, núm. 6, Estados Unidos: Stanford University.

Da Costa, Fernanda (2020), "Morte de trabalhadora doméstica por coronavírus escancara falta de políticas para proteger a clase", en Jornal da Universidade. Disponible en: https://www.ufrgs.br/jornal/morte-de-trabalhadora-domestica-por-coronavirusescancara-falta-de-politicas-para-proteger-a-classe/ [20 de junio de 2020].

Dalla Costa, Mariarosa y James, Selma (1972), El poder de la mujer y la subversión de la comunidad, México: Siglo XXI.

De la Fuente, María (2015), “Ideas de poder en la teoría feminista”, en Revista Española de Ciencia Política, vol. 39, España: Asociación Española de Ciencia Política y de Administración.

Del Campo, Andrea y Ruiz, Soledad (2013), "Empowerment of Unionised Household Workers”, en Psykhe, núm. 1, Chile: Pontificia Universidad Católica de Chile.

Delphy, Christine (1982), Por un feminismo materialista. El Enemigo principal y otros textos, España: LaSal.

Desimone, Rocío y Guerreo, Romina (2020) “'Acá encerradas en casa”. Empleadas domésticas en tiempo de coronavirus”, en Quépasa Web. Disponible en: https://www.quepasaweb. com.ar/aca-encerradas-en-casa-empleadas-domesticas-en-tiempo-decoronavirus/?fbc lid=IwAR0yMHy1psUPPkqw1uTHTCV7vGTVl6uP_xtnXKzPSBvL8ptJHLEN_ zuZj4 [20 de junio de 2020].

Federici, Silvia (2012), Revolution at Point Zero: Housework, Reproduction, and Feminist Struggle, Estados Unidos: Common Notions y PM Press.

Femenías, María Luis (2000), Sobre sujeto y género (Lecturas feministas desde Beauvoir a Butler), Argentina: Catálogos.

Fernández, Rosario (2017), “Trabajo doméstico pagado: la 'solución perfecta’ para la 'familia feliz' en Chile”, en Pavez, Jorge [ed.], (Des) Orden de género. Políticas y mercados del cuerpo en Chile, Chile: CRANN Editores.

Fernández, Rosario (2018a), "Commodification of domestic labour, the culture of servitude and the making of the Chilean nation”, en Österreichische Zeitschrift für Soziologie, núm. 43, Austria: Sociedad Austriaca de Sociología. 
Fernández, Rosario (2018b), "Performativity and Intimacy in Paid Domestic Work: Negotiating the Reproduction of Difference in Chile", Tesis de Doctorado en Sociología, Goldsmiths, University of London, Inglaterra.

Fernández, Rosario (2019), "El cerco y sus irrupciones: lecturas a partir del trabajo doméstico pagado", en Vera, Antonieta, Aguilera, Isabel y Fernández, Rosario [coords.], Nación, Otredad, Deseo: Producción de la diferencia en tiempos multiculturales, Chile: Universidad Academia de Humanismo Cristiano Ediciones.

Firestone, Shulamith (1970), The Dialectic of Sex: The Case for Feminist Revolution, Estados Unidos: William Morrow and Company.

Foucault, Michel (1999), La arqueología del saber, México: Siglo XXI.

Fraser, Myriam y Butler, Judith (2017), ¿ Reconocimiento o redistribución? Un debate entre marxismo y feminismo, España: Traficantes de Sueños.

Frye, Marilyn (1983), The Politics of Reality: Essays in Feminist Theory, Estados Unidos: The Crossing Press.

Gadamer, Hans-George (1977), Verdad y método I. Fundamentos de una hermenéutica filosófica, España: Sígueme.

Gálvez, Thelma y Todaro, Rosalba (1985) Yo trabajo asi ... en casa particular, Chile: Centro de Estudios de la Mujer.

Gálvez, Thelma y Todaro, Rosalba (1987), Trabajo doméstico remunerado. Conceptos, hechos, datos, Chile: Centro de Estudios de la Mujer.

Gálvez, Thelma y Todaro, Rosalba (1989), "Housework for pay in Chile: not just another job", en Chaney, Elsa y García Castro, Mary [eds.], Muchachas no more. Household workers in Latin America and the Caribbean, Estados Unidos: Temple University Press.

Gargallo, Francesca (2014), Feminismos desde Abya Yala. Ideas y proposiciones de las mujeres de 607 pueblos en nuestra América, México: Editorial Corte y Confección.

Glenn, Evelyn (1992), "From Servitude to Service Work: Historical Continuities in the Racial Division of Paid Reproductive Labor", en Signs, núm. 1, Estados Unidos: University of Chicago Press.

Gómez, Verónica y Jiménez, Andrés (2015), "Corresponsabilidad familiar y el equilibrio trabajo-familia: medios para mejorar la equidad de género”, en Polis, vol. 14, núm. 40, Chile: Universidad Bolivariana.

Gutiérrez, Encarnación (2010), Migration, domestic work and affect: a decolonial approach on value and the feminization of labor, Reino Unido: Routledge.

Gutiérrez, Encarnación y Brites, Jurema (2014), "Special Issue on Domestic Work between Regulation and Intimacy”, en Women's Studies International Forum, Holanda: Elsevier.

Hanrahan, Rebecca y Antony, Louise (2005), "Because I said so: Towards a feminist theory of authority", en Hypatia: A Journal of Feminist Philosophy, vol. 20, núm. 4, Estados Unidos: University of Oregon.

Herrera, Gioconda (2005), "Work and social reproduction in the lives of Ecuadorian domestic workers in Madrid", artículo para International Conference on Migration and Domestic Work in Global Perspective, del Netherlands Institute for Advanced Studies, realizado del 26 al 29 de mayo de 2005, en Wassenaar, Holanda.

Hill Collins, Patricia (2000), Black Feminist Thought: Knowledge, Consciousness, and the Politics of Empowerment, Estados Unidos: Routledge.

Hill Collins, Patricia y Bilge, Sirma (2016), Intersectionality, Reino Unido: Polity. 
Hoagland, Sarah y Frye, Marilyn (2000), Interpretaciones feministas de Mary Daly. Releyendo el cañón, Estados Unidos: Pennsylvania State University.

Hutchinson, Elizabeth (2006), Labores propias de su sexo, Chile: LOM.

Illanes, María Angélica (2006), Cuerpo y sangre de la política. La construcción histórica de las visitadoras sociales (1887-1940), Chile: LOM.

Irigaray, Luce (2009), Ese sexo que no es uno, España: Akal.

Jelin, Elizabeth (1976), Migración a las ciudades y participación en la fuerza de trabajo de las mujeres latinoamericanas: el caso del servicio doméstico, Argentina: Centro de Estudios de Estado y Sociedad.

King, Deborah (1988), "Multiple Jeopardy, Multiple Consciousness: The Context of a Black Feminist Ideology”, en Signs, vol. 14, núm. 1, Estados Unidos: University of Chicago Press.

Kojève, Alexandre (2005), La noción de autoridad, Argentina: Nueva Visión.

Leiva, Sandra (2015), "Organización social del cuidado en Bolivia y Chile: Estado y cuidadanía”, en Revista Austral de Ciencias Sociales, vol. 28, Chile: Universidad Austral de Chile.

León, Magdalena de (1997), Poder y empoderamiento de las mujeres, Colombia: Tercer Mundo Editores.

Lloyd, Moya (2005), Beyond identity politics feminism, power \& politics, Inglaterra: Sage.

MacKinnon, Catharine (1989), Toward a Feminist Theory of the State, Estados Unidos: Harvard University Press.

Martínez, Javier y Palacios, Margarita (2001), Encuesta Nacional. Liberalismo y conservadurismo en Chile. Análisis sobre opiniones y actitudes de las mujeres chilenas al fin del siglo $X X$, Chile: Grupo de Iniciativa Mujeres.

McNay, Lois (2016), "Agency”, en Disch, Lisa y Hawkesworth, Mary [eds.], The Oxford Handbook of Feminist Theory, Inglaterra: Oxford University Press.

Millett, Kate (1970), Politica sexual, España: Cátedra.

Mohanty, Chandra (2003), “Under Western Eyes” Revisited: Feminist Solidarity Through Anticapitalist Struggles”, en Signs, núm. 2, Estados Unidos: University of Chicago Press.

Montecino, Sonia (1993), Diagnóstico sobre inserción laboral de mujeres mapuches rurales y urbanas, Chile: Universidad de Chile, Facultad de Ciencias Sociales, Programa Interdisciplinario de Estudios de Género y Servicio Nacional de la Mujer.

Moreno, Aida (1989), "History of the Household Workers' movement in Chile, 19261983”, en Chaney, Elsa y García Castro, Mary [eds.], Muchachas no more. Household workers in Latin America and the Caribbean, Estados Unidos: Temple University Press.

Muraro, Luisa (1991), L'ordine simbolico della madre, Italia: Riuniti.

Nussbaum, Martha (1999), Sex and Social Justice, Inglaterra: Oxford University Press.

Okin, Susan (1989), Justice, Gender and the Family, Estados Unidos: Basic Books.

Oksala, Johanna (2016), Feminist Experiences: Foucauldian and Phenomenological Investigations, Estados Unidos: Northwestern University Press.

Pateman, Carole (1988), The sexual contract, Estados Unidos: Stanford University Press.

Precarias a la Deriva (2004), A la deriva por los circuitos de la precariedad femenina, España: Traficantes de Sueños.

Reich, Michelle (2017), Vivir sin nana, Chile: Lulu.com 
Rossi, Julia y Campanella, Lucía (2018), Los de abajo: tres siglos de sirvientes en el arte y la literatura de América Latina, Argentina: Universidad Nacional de Rosario.

Safiotti, Heleieth_(1978), Emprego Domestico e Capitalismo, Brasil: Vozes.

Saldaña, Abril (2014), "Tlazolteotl: The Filth Deity and the sexualization of paid domestic workers in Mexico", en Sexualities, núm. 1-2, Estados Unidos: Sage.

Seccombe, Wally (1974), “The housewife and her labor under capitalism”, en New Left Review, núm. 83, Inglaterra: New Left Review.

Souza, Julia (1980), "Paid Domestic Service in Brazil”, en Latin American Perspectives, núm. 7, Estados Unidos: Latin American Perspectives.

Staab, Silke y Maher, Kristen (2005), "Nanny Politics. The dilemmas of working women's empowerment in Santiago, Chile", en International Feminist Journal of Politics, núm. 1, Inglaterra: Taylor and Francis.

Staab, Silke y Maher, Kristen (2006), “The dual discourse about Peruvian domestic workers in Santiago de Chile: class, race, and a nationalist Project”, en Latin American Politics and Society, núm. 1, Inglaterra: Cambridge University Press.

Stefoni, Carolina (2002), "Mujeres inmigrantes peruanas en Chile”, en Papeles de Población, núm. 33, México: Universidad Autónoma del Estado de México.

Stefoni, Carolina y Fernández, Rosario (2011), "Mujeres inmigrantes en el trabajo doméstico: entre el servilismo y los derechos”, en Stefoni, Carolina [ed.], Mujeres inmigrantes en Chile. ¿Mano de obra o trabajadoras con derechos?, Chile: Universidad Alberto Hurtado.

Tinsman, Heidi (1992), “The Indispensable Services of Sisters: Considering Domestic Service in United States and Latin American Studies", en Journal of Women's History, vol. 4, núm. 1, Estados Unidos: Johns Hopkins University Press.

Valdés, Teresa y Valdés, Ximena [eds.] (2005), Familia y vida privada ¿Transformaciones, tensiones, resistencias o nuevos desafíos?, Chile: Centro de Estudios para el Desarrollo de la Mujer y Facultad Latinoamericana de Ciencias Sociales.

Valdés, Ximena (2006), La vida en común. Familia y vida privada en Chile y el medio rural en la segunda mitad del siglo XX, Chile: LOM.

Valdés, Ximena (2008), "Notas sobre la metamorfosis de la familia en Chile”, en Arriagada, Irma [ed.], Futuro de las familias y desafíos para las politicas públicas, Chile: División de Desarrollo Social, Comisión Económica para América Latina y el Caribe.

Valenzuela, María Elena y Mora, Claudia [eds.] (2009), Trabajo doméstico: un largo camino hacia el trabajo decente, Chile: Organización Internacional del Trabajo.

Valenzuela, Mariana (2019), "La figura de la madre en los casos de las presidentas latinoamericanas Michelle Bachelet (Chile), Cristina Fernández (Argentina) y Dilma Rousseff (Brasil)", en Revista de Estudios Sociales, núm. 69, Colombia: Universidad de Los Andes.

Vera, Antonieta (2016), “La superioridad moral de la mujer”: sobre la norma racializada de la femineidad en Chile”, en Historia y Politica, núm. 36, Chile: Universidad Católica del Maule.

Verdú, Ana (2012), "La desaparición de las diosas como metáfora de la pérdida de autoridad de las mujeres", en Revista Feminismo/s, núm. 20, España: Universidad de Alicante.

Weber, Max (1964), Economía y sociedad, México: Fondo de Cultura Económica.

Young, Iris Marion (1992), "Five Faces of Oppression", en Wartenberg, Thomas [ed.], Rethinking Power, Estados Unidos: SUNY Press. 
Rosario Fernández Ossandón. Doctora en Sociología por Goldsmiths Universidad de Londres, investigadora posdoctoral Instituto de Estudios Avanzados, Universidad Santiago de Chile, Centro Núcleo Milenio Autoridad y Asimetrías de Poder / Millennium Nucleus Center Authority and Power Asymmetries, Chile. Investigadora Principal proyecto Fondecyt Postdoctoral núm. 3210057, financiado por la Agencia Nacional de Investigación y Desarrollo de Chile (2021-2024). Líneas de investigación: género y feminismo; familia, intimidad y política; trabajo doméstico; afectos; memorias y archivos. Publicaciones recientes: Fernández, Rosario y Moreno,Claudia(2019), “Feminismosenlasrevueltas”,en Araujo,Kathya[ed.], Hilos Tensados. Para leer el octubre chileno, Chile: Instituto de Estudios Avanzados, Universidad de Santiago de Chile. Fernández, Rosario (2019), "El cerco y sus irrupciones: lecturas a partir del trabajo doméstico pagado", en Vera, Antonieta, Aguilera, Isabel y Fernández, Rosario, Nación Otredad, Deseo: Producción de la diferencia en tiempos multiculturales, Chile: Universidad Academia de Humanismo Cristiano Ediciones. Fernández, Rosario (2018), "Commodification of domestic labour, the culture of servitude and the making of the Chilean nation", en Österreichische Zeitschrift für Soziologie, vol. 43, Austria: Sociedad Austriaca de Sociología. Indexación Scopus. https://doi.org/10.1007/s11614-018-0290-1. 